\title{
Enmienda con compost y fertilización de suelos afectados por incendios: Respuesta de dos especies nativas de la Patagonia bajo condiciones de invernadero
}

\author{
Marina Gonzalez-Polo ${ }^{\varpi}$; Mariana L. Agüero \& Elisa Castán \\ INIBIOMA, CONICET-Universidad Nacional Comahue. San Carlos de Bariloche, Río Negro, Argentina.
}

\begin{abstract}
RESUmen. Las actividades humanas alteraron la frecuencia y la intensidad de los incendios, lo cual puede tener consecuencias severas sobre los ecosistemas terrestres. Se sugiere que aplicar enmiendas y trasplantar especies nativas serían alternativas exitosas para recuperar zonas incendiadas. En la región andina de la Patagonia Argentina se produce compost de biosólidos que se podría usar a tal fin. Sin embargo, se conoce poco sobre el efecto de estas enmiendas orgánicas en el crecimiento de las plantas nativas. El objetivo del trabajo fue evaluar la respuesta al agregado de compost y la fertilización nitrogenada de dos especies nativas: ciprés (Austrocedrus chilensis) y roble (Nothofagus obliqua), cultivadas en un sustrato volcánico forestal afectado por un incendio. Después de 4 años se observó que el efecto de los tratamientos (en la planta y en el suelo) dependió de la especie. El efecto principal del compost fue un aumento marcado en la disponibilidad de fósforo en los suelos. El roble incrementó su biomasa y modificó parámetros arquitecturales en respuesta a la fertilización nitrogenada, pero no presentó cambios ante la adición de compost. El ciprés, por el contrario, no respondió a la fertilización nitrogenada, pero sí incrementó su biomasa en el tratamiento con compost y fertilización. Es posible que agregar nitrógeno haya favorecido la absorción de nutrientes que aporta el compost, como fósforo, calcio y magnesio.
\end{abstract}

[Palabras clave: Nothofagus obliqua, Astrocedrus chilensis, compost de biosólidos, fuego]

\begin{abstract}
Aвstract. Burnt soils treated with compost and N fertilizer: effects on seedlings of two native species to Andean-Patagonia. Human activities have altered the frequency and intensity of wildfires, which can have severe consequences on forest ecosystems. Organic amendments and transplantation of native species are suggested as successful restoration alternatives for burnt areas. Biosolids composts produced in the Andean Patagonian region of Argentina could be used for this purpose, but little is known about the effects of organic amendments on seedling growth of native species. The objective of this study was to evaluate the response of the native species Austrocedrus chilensis and Nothofagus obliqua the addition of compost and/or nitrogen fertilizer in a greenhouse experiment using a volcanic forest soil affected by fire. After 4 years, treatment effects on plant growth and soil characteristics depended on the species. The main effect of biosolids compost on soil fertility was a marked increase in the availability of P from the soils of the two species. N. obliqua biomass increased and changed architectural parameters in response to nitrogen fertilization, but did not show changes due to compost addition. On the other hand, A. chilensis did not respond to $\mathrm{N}$ fertilization, but increase its biomass with compost and fertilization. Possibly $\mathrm{N}$ addition favored the nutrients uptake, such as phosphorus, calcium and magnesium provided by the compost.
\end{abstract}

[Keywords: Nothofagus obliqua, Astrocedrus chilensis, biosolid compost, fire]

\section{INTRODUCCIÓN}

Los incendios naturales son importantes fuerzas ecológicas y evolutivas. En muchos ecosistemas, el fuego regula la estructura y composición de las comunidades vegetales (Biganzoli et al. 2009; Torres Curth et al. 2012). Sin embargo, las actividades humanas han alterado la frecuencia y la intensidad de los incendios (Di Bella et al. 2006), y estos cambios pueden afectar de forma negativa los ecosistemas. La materia orgánica del suelo tiene muchas funciones ecológicas y suele ser afectada durante los incendios. Entre sus funciones principales, mejora la estructura del suelo y la actividad microbiana y, por lo tanto, regula la dinámica y la provisión de

Editora asociada: María Semmartin 西 marina.gonzalezpolo@comahue-conicet.gob.ar agua y nutrientes para el crecimiento vegetal. El nitrógeno es el principal nutriente que limita el crecimiento vegetal en ecosistemas terrestres (Schlesinger 1997; LeBauer and Treseder 2008), y su mayor reservorio en el suelo es la materia orgánica. Por lo tanto, las pérdidas de materia orgánica y nitrógeno durante los incendios afectarán en mayor o menor grado el funcionamiento a posteriori del ecosistema. El efecto del fuego sobre la materia orgánica y los nutrientes del suelo depende, principalmente, de su intensidad. El carbono y el nitrógeno son muy afectados porque comienzan a perderse en formas gaseosas a temperaturas relativamente bajas (200-350 $\left.{ }^{\circ} \mathrm{C}\right)$; en cambio, durante los incendios se produce una "fertilización" del suelo con

Recibido: 24 de Abril de 2019

Aceptado: 2 de Junio de 2020 
nutrientes poco volátiles (fósforo y cationes) $\mathrm{y}$ un aumento del $\mathrm{pH}$ por efecto de cationes básicos, y también la pérdida de materia orgánica acidificante (Urretavizcaya 2010; La Manna and Barroetaveña 2011).

El fuego es uno de los disturbios más importantes en la Región Andino-Patagónica. Desde el establecimiento de las poblaciones europeas ha tenido lugar una perturbación del régimen de fuego natural en la Patagonia, con un aumento de la frecuencia en los bosques del oeste y una disminución en las zonas áridas (Veblen et al. 1992). Los registros para el NO de la Patagonia Argentina indican que entre 1973-2011, los incendios afectaron cerca de 56000 ha (Oddi 2013). El último gran incendio forestal se produjo en el verano de 2015 y abarcó 30000 ha en las cercanías de Esquel. En suelos arenosos de la estepa arbustiva se observó una disminución de más del 50\% del C orgánico del suelo diez meses después de un incendio (Kowaljow and Mazzarino 2007). Más aun, en suelos volcánicos, a pesar de su alta capacidad para estabilizar y proteger la materia orgánica, también se observaron pérdidas de materia orgánica que, en algunos casos, implicaron una disminución del 65\% del carbono orgánico y del 30\% del nitrógeno total (Alauzis et al. 2004; Kitzberger et al. 2005; Morales et al. 2013).

Dado que los incendios afectan fundamentalmente a la fracción orgánica del suelo, la recuperación de la fertilidad depende de que se recupere la materia orgánica. Una alternativa para la rehabilitación post-fuego es aplicar enmiendas orgánicas (Cordovil et al. 2011; Cellier et al. 2012). En la región se producen diferentes tipos de enmiendas orgánicas que se podrían usar para la recuperar suelos quemados, lo que les aportaría materia orgánica y los protegería de la erosión. En particular, los compost de origen animal, como estiércoles y lodos cloacales, presentan una concentración alta de carbono, nitrógeno y fósforo (Cooperband and Middleton 1996; Tognetti et al. 2005). Por otro lado, estudios en bosques de Austrocedrus chilensis y Nothofagus pumilio muestran que la expresión del banco de semillas no sería una estrategia viable de revegetación de los sitios quemados (Gobbi and Sancholuz 1992; Urretavizcaya and Defossé 2004; Varela et al. 2006; Urretavizcaya et al. 2018). En especial, Varela et al. (2006) sugieren aplicar enmiendas y trasplantar plantines de especies nativas como alternativa para recuperar zonas afectadas por el fuego.
El estrato arbóreo de los bosques andinopatagónicos está dominado por especies del género Nothofagus (representan el $80 \%$ ) y por la conífera Austrocedrus chilensis (Veblen et al. 1996; Ayesa et al. 2002). Nothofagus obliqua y A. chilensis son dos de las especies arbóreas patagónicas de mayor potencial forestal por sus características de desarrollo y la calidad de su madera. Para reinstalar estas especies en áreas degradadas y establecer rodales con fines paisajísticos o para explotación maderera es necesario conocer el efecto de las enmiendas orgánicas (en particular, compost de biosólidos, caracterizado por altos contenidos de macronutrientes) en el crecimiento y la nutrición de estas plantas. El objetivo del presente trabajo fue evaluar la respuesta de dos especies arbóreas nativas de los bosques andino-patagónicos (i.e., roble pellín: Nothofagus obliqua y ciprés: Austrocedrus chilensis) al agregado de compost y fertilización nitrogenada en un ensayo de invernadero utilizando un suelo volcánico forestal afectado por un incendio.

\section{Materiales y Métodos}

\section{Diseño experimental}

Los suelos fueron colectados de un rodal monoespecífico de ciprés de la cordillera en el extremo más seco de su distribución. Los suelos son clasificados como Udivitrands y fueron afectados por un incendio severo 2 años previos al muestreo. Los suelos quemados utilizados en el ensayo tenían aproximadamente 1.6 veces menos materia orgánica (medida como \% de C) que los suelos cercanos no afectados por el incendio.

En macetas de $250 \mathrm{~mL}$ (14 cm de largo por $6.5 \mathrm{~cm}$ de ancho) se repicaron 42 plantines de ciprés de la cordillera (Astrocedrus chilensis) y 42 plantines de roble pellín (Nothofagus obliqua) de 1 mes de edad (una plántula por maceta). Cada maceta se llenó con una mezcla 50:50 (v: v) de suelo quemado (tamizado por una malla de $5 \mathrm{~mm}$ ) y turba de Carex sp., para evitar la compactación del suelo en las macetas; dada la diferencia de densidad, el suelo representó en peso $89 \%$ de la mezcla. A la mitad se les agregó compost de biosólidos en una dosis de $4 \mathrm{~g}$ de compost seco por cada $100 \mathrm{~g}$ de sustrato seco. La otra mitad se mantuvo como control. El compost se obtuvo de la planta de compostaje de la ciudad de Bariloche utilizando chips de poda como agente estructurante y su composición varía en función del tiempo de 
almacenamiento a campo. Las características del compost utilizado en el experimento se indican en la Tabla 1.

Durante la primera estación de crecimiento, todas las plántulas crecieron sin fertilización inorgánica y en la segunda estación de crecimiento, la mitad de ellas fueron fertilizadas con nitrógeno. Se utilizó urea en una dosis equivalente a $100 \mathrm{mg} \mathrm{N} / \mathrm{kg}$ de suelo en el caso del ciprés, y de $200 \mathrm{mg} \mathrm{N} / \mathrm{kg}$ de suelo en el ensayo con roble. Se aplicaron dosis distintas en ambas especies ya que estudios previos mostraban que dosis mayores afectan la supervivencia de los plantines de ciprés. El fertilizante se fraccionó en tres aplicaciones durante la estación de crecimiento. El diseño experimental fue un factorial completo, con adición de compost y fertilización nitrogenada como factores. Las plantas fueron cosechadas 4 años luego de ser repicadas. Se aplicó riego durante todo el ensayo, con frecuencia diaria (o cada 2 días) durante la primavera-verano y una vez por semana en otoño-invierno.

\section{Variables de respuesta}

Las plantas se cosecharon luego de 4 años después de repicadas. Se registró la longitud aérea, el diámetro basal y el número de nudos de todos los individuos. Se registró el diámetro de la raíz en el punto de unión con el vástago. Además, en el caso del ciprés se registró el número de ramas, y en el caso del roble, la longitud y la posición relativa de la hoja mayor. Finalmente, la biomasa se secó a 70 ${ }^{\circ} \mathrm{C}$ durante 48 horas y se registró el peso seco aéreo (vástago+hojas) y subterráneo (raíces).

En tres repeticiones de tejido foliar por tratamiento (cada una compuesta por 7 plantines) se determinó C y nutrientes. El

Tabla 1. Características químicas del compost de biosólidos utilizado en el ensayo.

Table 1. Chemical characteristics of biosolids compost employed in the greenhouse trial.

\begin{tabular}{lc}
\hline & $\begin{array}{c}\text { Compost de } \\
\text { Biosólidos }\end{array}$ \\
\hline $\mathrm{pH}$ & 5.4 \\
Conductividad eléctrica $(\mathrm{dS} / \mathrm{m})$ & 0.21 \\
Nitrógeno $(\mathrm{mg} / \mathrm{kg})$ & 8.7 \\
$\mathrm{C}$ orgánico $(\mathrm{g} / \mathrm{kg})$ & 135.9 \\
$\mathrm{P}$ extractable $(\mathrm{mg} / \mathrm{kg})$ & 245.2 \\
$\mathrm{P}$ total $(\mathrm{g} / \mathrm{kg})$ & 3.7 \\
$\mathrm{Ca}(\mathrm{g} / \mathrm{kg})$ & 6.5 \\
$\mathrm{Mg}(\mathrm{g} / \mathrm{kg})$ & 4.4 \\
$\mathrm{~K}(\mathrm{~g} / \mathrm{kg})$ & 1.3 \\
$\mathrm{Na}(\mathrm{g} / \mathrm{kg})$ & 0.3 \\
\hline
\end{tabular}

carbono y el nitrógeno se analizaron con un analizador elemental de C-N (Thermo Electron Corporation Flash EA 1112). El fósforo y los cationes se analizaron por medio de digestiones $550{ }^{\circ} \mathrm{C}$ y se extrajeron con $\mathrm{HCl}$ concentrado. El fósforo se determinó por el método del molibdato-ácido ascórbico y los cationes (K, Ca y Mg) por espectrometría de absorción atómica.

Una vez cosechadas las plantas, se tomaron muestras del suelo contenido en las macetas y se analizó su conductividad eléctrica y su pH en agua (1:5 y 1:2.5 suelo:agua, respectivamente); $\mathrm{C}$ y $\mathrm{N}$ total, con el analizador elemental ya mencionado y $\mathrm{P}$ extractable ( $\mathrm{P}$ Olsen) en $\mathrm{NaHCO}_{3}$ 0.5 M (1:20 suelo:solución) y determinado por el método de molibdato ácido ascórbico (Kuo 1996).

\section{Análisis estadístico}

Para analizar el efecto del compost, la fertilización y sus interacciones se utilizaron ANOVA de dos vías, y la comparación de las medias se realizó con la prueba de Tukey. Los datos fueron transformados para cumplir con los supuestos de normalidad y homocedasticidad. Los análisis estadísticos se realizaron usando Statistica 7 software (StatSoft).

\section{Resultados}

\section{Características químicas del suelo}

Los tratamientos aumentaron de manera pequeña, pero significativa, el $\mathrm{pH}$ y la conductividad del suelo (Tabla 2). El C y el N total de los suelos de macetas sembradas con roble aumentaron con la adición de compost, mientras que en el caso del ciprés aumentaron con la fertilización. Finalmente, el P extractable del suelo mostró un aumento significativo con el agregado de compost en el suelo de ambas especies (Tabla 2).

\section{Biomasa vegetal y concentración de nutrientes en hojas}

El efecto de la fertilización en conjunto con el agregado de compost aumentó la biomasa total de ciprés (Figura 1 A). Sin embargo, la biomasa del roble sólo se incrementó con la fertilización, independientemente del agregado de compost (Figura 1B).

El C total y los nutrientes en hojas fueron afectados por los tratamientos (Tabla 3). El C total en las hojas de ciprés disminuyó 
Tabla 2. Características químicas del suelo en los distintos tratamientos al final del experimento. Sólo se indican diferencias significativas entre medias (prueba de Tukey, $P<0.05$ ) cuando la interacción fue significativa; en caso de interacción no significativa, los factores significativos se indican en la nota al pie del cuadro. El error estándar se indica entre paréntesis $(n=3)$.

Table 2. Soil chemical characteristics at the end of the experiment. Only significant differences between means are indicated (Tukey test, $P<0.05$ ) when the interaction was significant, in case of non-significant interaction the significant factors are indicated at the footnote. Standard errors of means are given in parentheses $(n=3)$.

\begin{tabular}{llccccc}
\hline Especie & Tratamiento & $\mathrm{pH}$ & $\begin{array}{c}\text { Conductividad } \\
\text { eléctrica } \\
(\mathrm{dS} / \mathrm{m})\end{array}$ & $\begin{array}{c}\text { C Total } \\
(\mathrm{g} / \mathrm{kg})\end{array}$ & $\begin{array}{c}\mathrm{N} \text { Total } \\
(\mathrm{g} / \mathrm{kg})\end{array}$ & $\begin{array}{c}\text { P Extractable } \\
(\mathrm{mg} / \mathrm{kg})\end{array}$ \\
\hline Ciprés & Control & $6.7(0.03) \mathrm{a}$ & $0.04(0.002) \mathrm{a}$ & $51.3(11.00)$ & $2.6(0.07)$ & $2.8(0.21) \mathrm{c}$ \\
& Fertilización & $6.8(0.02) \mathrm{a}$ & $0.05(0.003) \mathrm{a}$ & $63.3(7.80)$ & $3.0(0.20)$ & $2.7(0.06) \mathrm{c}$ \\
& Compost & $6.8(0.01) \mathrm{a}$ & $0.05(0.0008) \mathrm{a}$ & $55.8(4.77)$ & $2.8(0.15)$ & $8.5(0.36) \mathrm{b}$ \\
& Fertilización+Compost & $6.7(0.02) \mathrm{a}$ & $0.05(0.0006) \mathrm{a}$ & $65.3(2.29)$ & $3.2(0.03)$ & $12.7(0.27) \mathrm{a}$ \\
Roble & Control & $6.6(0.02)$ & $0.06(0.001)$ & $53.6(4.31)$ & $2.6(0.11)$ & $2.8(0.10) \mathrm{b}$ \\
& Fertilización & $6.6(0.03)$ & $0.07(0.003)$ & $53.6(1.73)$ & $2.6(0.07)$ & $3.7(0.46) \mathrm{b}$ \\
& Compost & $6.8(0.02)$ & $0.05(0.002)$ & $74.3(4.48)$ & $3.4(0.07)$ & $11.0(0.21) \mathrm{a}$ \\
& Fertilización+Compost & $6.7(0.03)$ & $0.06(0.004)$ & $65.3(6.47)$ & $3.1(0.21)$ & $8.9(0.97) \mathrm{a}$ \\
\hline
\end{tabular}

Ciprés: C Total: $P_{\text {Fert }}<0.06 ; \mathrm{N}$ Total $P_{\text {Fert }}<0.05$. Roble: $\mathrm{pH}: P_{\text {Compost }}<0.01$; conductividad eléctrica: $P_{\text {Compost }}<0.05$, $P_{\text {Fert }}<0.001 ;$ C Total: $P_{\text {Compost }}<0.01 ; \mathrm{N}$ Total: $P_{\text {Compost }}<0.01$.
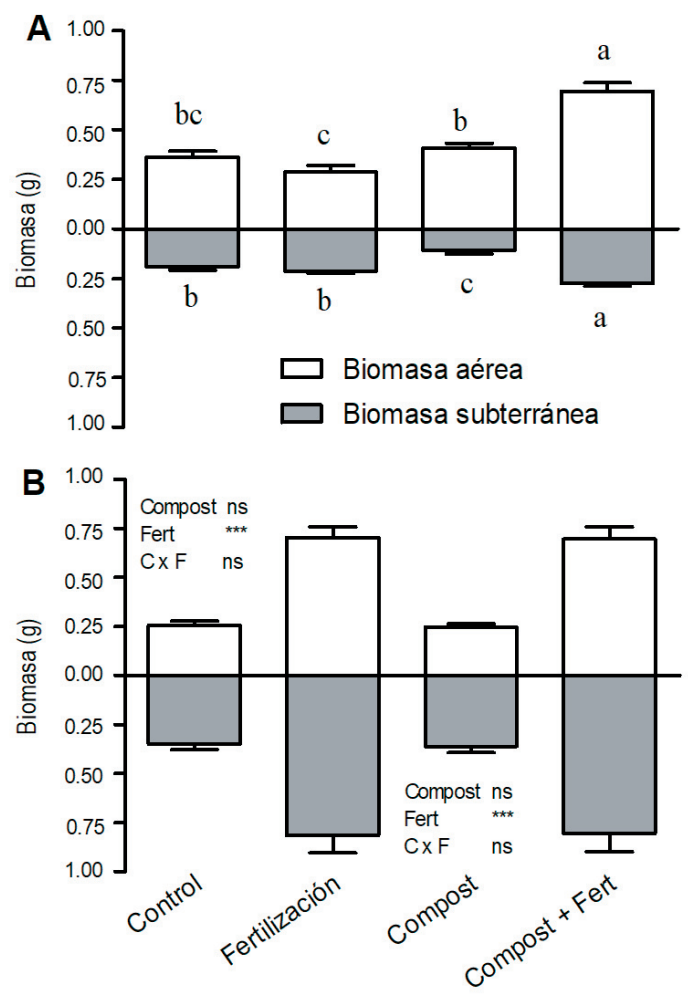

Figura 1. Efecto del compost y la fertilización con $\mathrm{N}$ en la biomasa aérea y subterránea de los plantines de: A) Ciprés y B) Roble. Se muestran los resultados del análisis de varianza de dos factores $\left({ }^{* * *} P<0.001\right)$, cuando la interacción entre los factores fue significativa $(P<0.05)$ se indican diferencias significativas entre medias según prueba de Tukey $(P<0.05)$.

Figure 1. Effect of compost and $\mathrm{N}$ fertilization on shoot and root biomass of A) Ciprés seedlings and B) Roble seedlings. Two-way ANOVA results are shown $\left({ }^{* * *} P<0.001\right)$, when the interaction between the factors was significant $(P<0.05)$, significant differences between means are indicated according to Tukey's test $(P<0.05)$. en $1.5 \%$ con el agregado de compost, independientemente de la fertilización, pero el compost aumentó el $\mathrm{N}$ y el $\mathrm{P}$ en hojas de esta especie en un $40 \%$ y un $60 \%$, respectivamente, respecto al control (Tabla 3 ).

Las plantas de ciprés que recibieron fertilización + compost acumularon más $\mathrm{P}, \mathrm{Ca}$, y Mg que el resto de los tratamientos (Tabla 4). Por el contrario, en el caso del roble, el efecto más marcado en los nutrientes correspondió a la fertilización (independientemente del agregado de compost), que aumentó en un $18 \%$ el contenido de $\mathrm{N}$ total en las hojas respecto al control (Tabla 3 ).

\section{Variables arquitecturales}

La longitud aérea de las plantines de ciprés aumentó respecto al control con la aplicación de todos los tratamientos (Figura 2). Los mayores diámetros basales y de la raíz correspondieron al tratamiento con agregado conjunto de compost y fertilizante. La longitud de la raíz principal y el número de ramas de ciprés aumentó con la fertilización. La relación biomasa aérea:biomasa radical bajo agregado de compost fue mayor que en el resto de los casos (2.9 \pm 0.14 compost vs. $2.2 \pm 0.05 \mathrm{sin}$ compost; $P<0.001)$.

A diferencia del ciprés, todas las variables arquitecturales medidas en roble respondieron fuertemente al agregado de fertilizante (Figura 3). La relación biomasa aérea:biomasa radical evidenció una menor asignación de recursos a la biomasa subterránea de las plantas fertilizadas (Figura 1B). 
Tabla 3. Concentraciones de nutrientes de las hojas verdes de ciprés y roble. Letras distintas indican diferencias significativas cuando la interacción fue significativa; los factores significativos se indican en la nota al pie del cuadro. Se combinaron 7 muestras por repetición. El error estándar se indica entre paréntesis $(n=3)$.

Table 3. Mean values of $\mathrm{C}, \mathrm{N}$ and $\mathrm{P}$ concentrations in green leaves with compost addition and $\mathrm{N}$ fertilization. Different letters indicate significant differences when the interaction was significant, the significant factors are indicated at the foot note. Standard errors of means are given in parentheses $(n=3)$.

\begin{tabular}{llccc}
\hline Especie & Tratamiento & $\begin{array}{c}\text { C total } \\
(\mathrm{g} / \mathrm{kg})\end{array}$ & $\begin{array}{c}\mathrm{N} \text { total } \\
(\mathrm{g} / \mathrm{kg})\end{array}$ & $\begin{array}{c}\text { P total } \\
(\mathrm{g} / \mathrm{kg})\end{array}$ \\
\hline Ciprés & Control & $470.9(0.42)$ & $8.5(0.12) \mathrm{b}$ & $1.1(0.06) \mathrm{c}$ \\
& Fertilización & $472.0(0.08)$ & $8.8(0.26) \mathrm{b}$ & $1.1(0.09) \mathrm{c}$ \\
& Compost & $462.7(1.99)$ & $13.9(0.06) \mathrm{a}$ & $2.7(0.08) \mathrm{a}$ \\
& Fertilización+Compost & $466.2(0.12)$ & $7.5(0.14) \mathrm{b}$ & $1.6(0.03) \mathrm{b}$ \\
& Control & $478.2(0.12) \mathrm{a}$ & $11.7(0.14)$ & - \\
& Fertilización & $469.9(0.59) \mathrm{bc}$ & $13.9(0.20)$ & - \\
& Compost & $471.9(0.01) \mathrm{b}$ & $11.3(0.03)$ & - \\
& Fertilización+Compost & $467.4(0.59) \mathrm{c}$ & $14.1(0.30)$ & - \\
\hline
\end{tabular}

Ciprés: C Total: $P_{\text {Compost }}<0.01, \mathrm{P}$ total: $P_{\text {Compost }}<0.0001$. Roble: $\mathrm{N}$ total: $P_{\text {Fert }}<0.0001$

\section{$\square$ - N Fertilización}

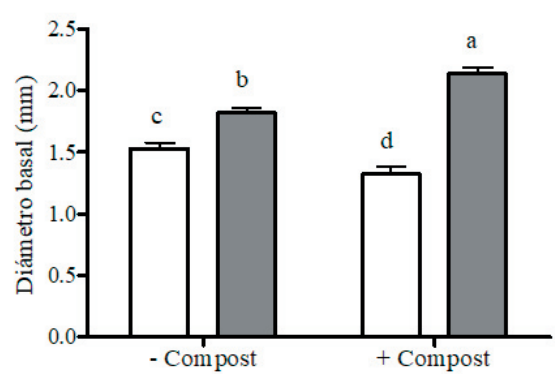

$\square+\mathrm{N}$ Fertilización
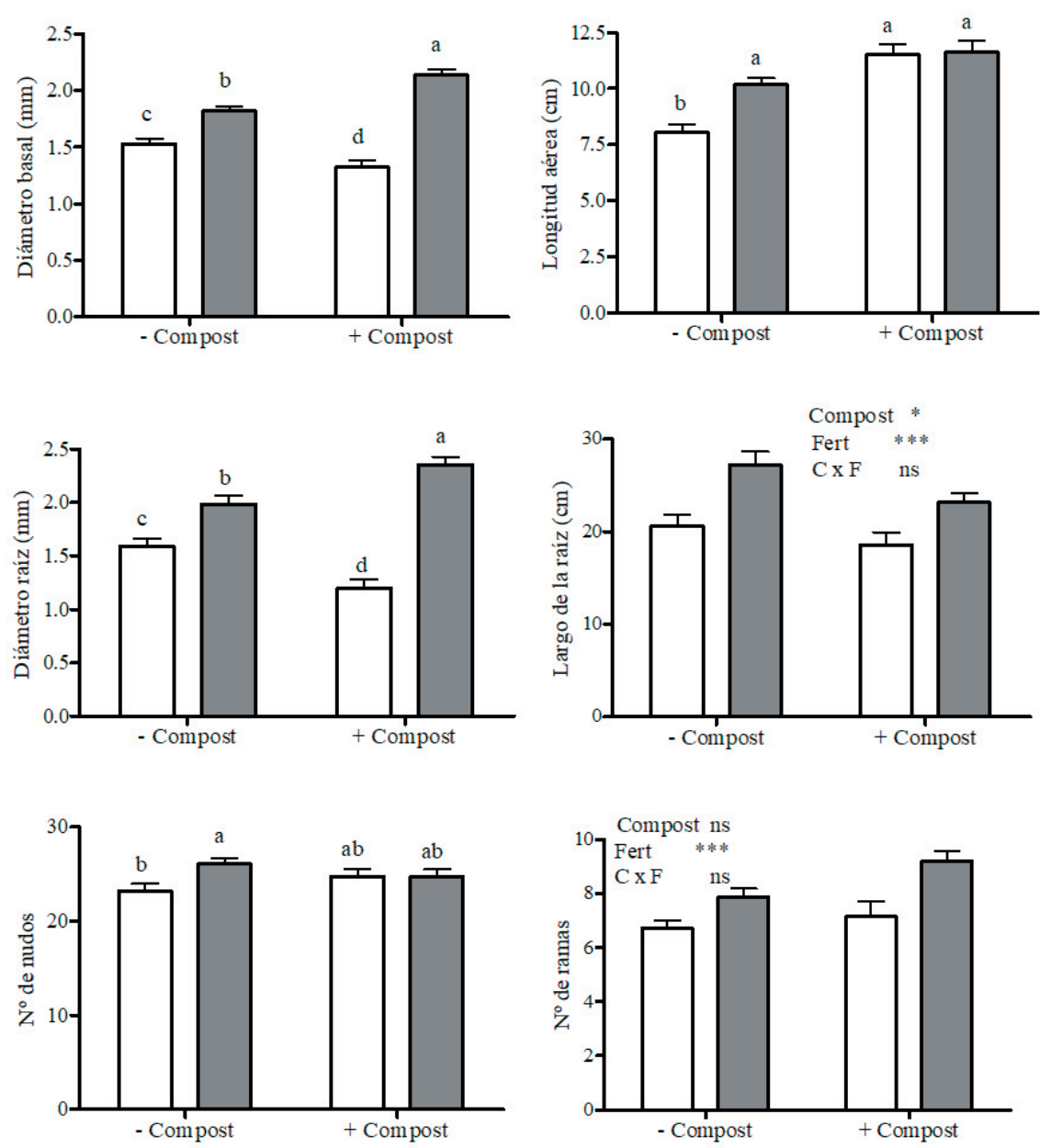

Figura 2. Efecto del compost y la fertilización con $\mathrm{N}$ sobre variables arquitecturales de ciprés. Se muestran los resultados del análisis de varianza de dos factores $\left({ }^{* * *} P<0.001 ;{ }^{*} P<0.05\right)$; cuando la interacción entre los factores fue significativa $(P<0.05)$, se indican diferencias significativas entre medias según prueba de Tukey $(P<0.05)$.

Figure 2. Effect of compost and $\mathrm{N}$ fertilization on the architectural variables of cypress seedlings. Two-way ANOVA results are shown $\left({ }^{* * *} P<0.001 ;{ }^{*} P<0.05\right)$; when the interaction between the factors was significant $(P<0.05)$, significant differences between means are indicated according to Tukey's test $(P<0.05)$. 
Tabla 4. Nutrientes acumulados en biomasa de ciprés (biomasa aérea por concentración de nutrientes). Letras distintas indican diferencias significativas cuando la interacción fue significativa, los factores significativos se indican en la nota al pie del cuadro. Se combinaron 7 muestras por repetición $(n=3)$. El error estándar se indica entre paréntesis $(n=3)$.

Table 4. Mean values of nutrient contents in green leaves (shoot biomass $x$ nutrient concentration) with compost addition and $\mathrm{N}$ fertilization. Different letters indicate significant differences when the interaction was significant, the significant factors are indicated at the foot note. Composite samples consisted of 7 seedlings each. Standard errors of means are given in parentheses; $n=3$ for all treatments.

\begin{tabular}{llccccc}
\hline Especie & Tratamiento & $\begin{array}{c}\mathrm{N} \\
(\mu \mathrm{g} / \mathrm{ind})\end{array}$ & $\begin{array}{c}\mathrm{P} \\
(\mu \mathrm{g} / \mathrm{ind})\end{array}$ & $\begin{array}{c}\mathrm{K} \\
(\mu \mathrm{g} / \mathrm{ind})\end{array}$ & $\begin{array}{c}\mathrm{Ca} \\
(\mu \mathrm{g} / \mathrm{ind})\end{array}$ & $\begin{array}{c}\mathrm{Mg} \\
(\mu \mathrm{g} / \mathrm{ind})\end{array}$ \\
\hline Ciprés & Control & $0.3(0.03)$ & $0.04(0.003) \mathrm{c}$ & $0.3(0.03) \mathrm{a}$ & $0.4(0.05) \mathrm{b}$ & $0.05(0.004) \mathrm{b}$ \\
& Fertilización & $0.4(0.02)$ & $0.04(0.002) \mathrm{c}$ & $0.3(0.01) \mathrm{a}$ & $0.5(0.05) \mathrm{b}$ & $0.05(0.004) \mathrm{b}$ \\
& Compost & $0.4(0.06)$ & $0.08(0.008) \mathrm{b}$ & $0.2(0.02) \mathrm{a}$ & $0.3(0.01) \mathrm{b}$ & $0.03(0.002) \mathrm{b}$ \\
& Fertilización+Compost & $0.5(0.04)$ & $0.12(0.010) \mathrm{a}$ & $0.4(0.11) \mathrm{a}$ & $0.8(0.10) \mathrm{a}$ & $0.09(0.010) \mathrm{a}$ \\
\hline
\end{tabular}

Ciprés: N en biomasa: $P_{\text {Compost }}<0.01$ y $P_{\text {Fert }}<0.05$

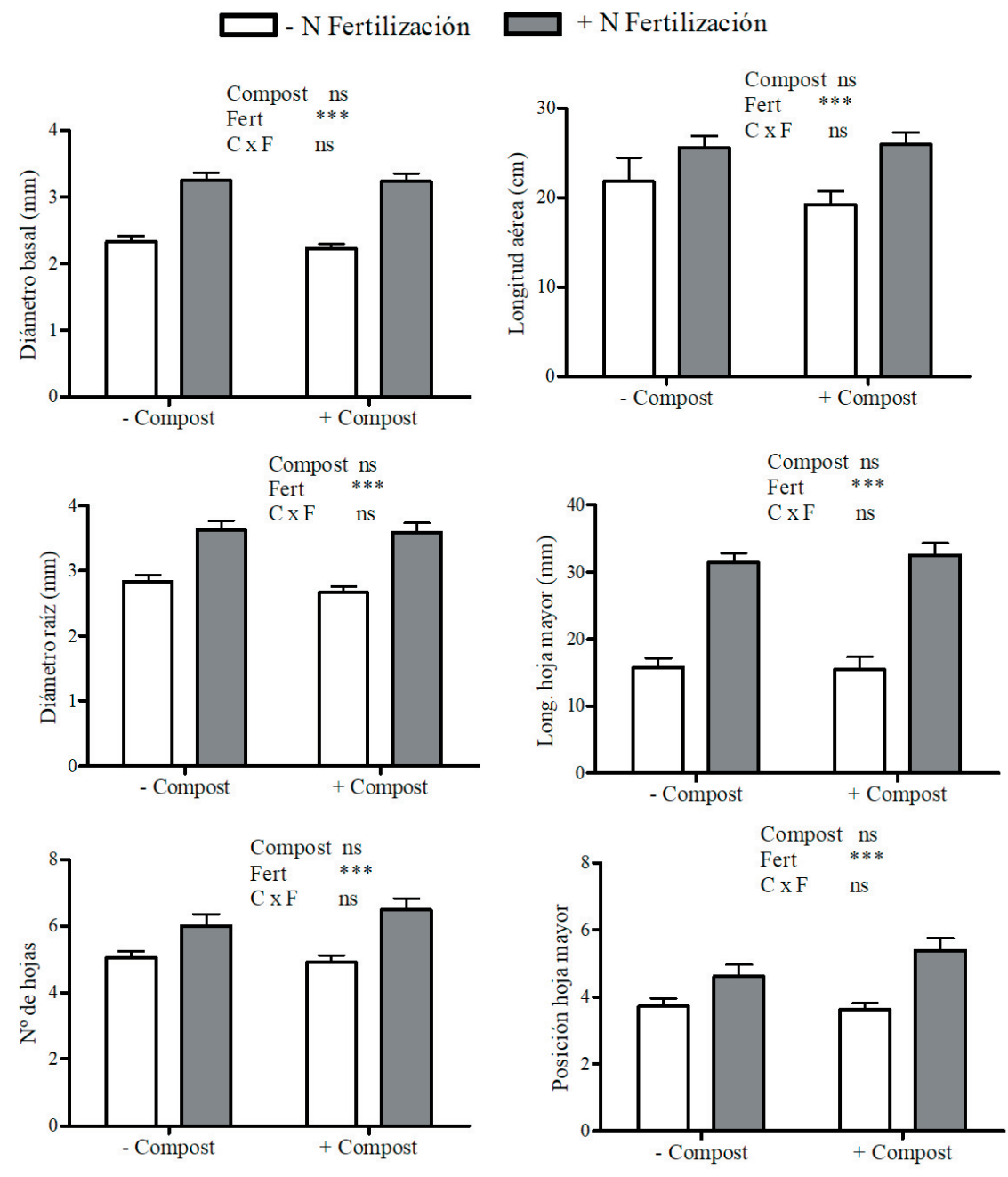

Figura 3. Efecto del compost y la fertilización con $\mathrm{N}$ sobre variables arquitecturales de roble. Se muestran los resultados del análisis de varianza de dos factores $\left({ }^{* * *} P<0.001\right)$.

Figure 3. Effect of compost and $\mathrm{N}$ fertilization on the architectural variables of roble seedlings. Two-way ANOVA results are shown $\left({ }^{* *} P<0.001\right)$. 


\section{Discusión}

Después de cuatro estaciones de crecimiento se observó un claro efecto residual del compost en la disponibilidad de $\mathrm{P}$ de los suelos de ambas especies. Si bien los incendios suelen provocar una fertilización con P disponible, en suelos volcánicos se observó que este P es nuevamente fijado por los alofanos entre 2 y 4 años después del incendio (Alauzis et al. 2004). Por lo tanto, el suelo control de los ensayos presentó valores muy bajos de P-Olsen, mucho más bajos que $10 \mathrm{mg} / \mathrm{kg}$, valor sugerido como umbral para la productividad de especies leñosas nativas en suelos volcánicos (Parfitt et al. 2005). Los compost de origen animal (i.e., estiércoles y biosólidos) son ricos en formas inorgánicas de $\mathrm{P}$ que aumentan su disponibilidad a largo plazo, incluso en suelos volcánicos como se observó en este trabajo. El aumento detectado no implica un riesgo de contaminación, ya que es cercano al valor crítico.

El efecto de la enmienda de compost sobre el suelo sólo se evidenció en las macetas sembradas con roble. El agregado de compost mejoró las concentraciones de $\mathrm{C}$ y $\mathrm{N}$ total del suelo. Por el contrario, luego de 4 años no se detectó un aumento de materia orgánica por el agregado de compost en las macetas sembradas con ciprés. Estos resultados ponen de relevancia la compleja interacción suelo-planta, en la que los exudados radicales podrían jugar un rol clave en determinar el efecto diferencial sobre el suelo de una misma enmienda. Cabe destacar que las dosis de compost aplicadas fueron bajas en comparación con otros estudios (Basil et al. 2009; Varela and Martínez 2013). Esto se debe a que las dosis que se usaron este trabajo equivalen a las que se pueden aplicar a campo, mientras que el objetivo de otros estudios es usar compost como sustrato para producir plantines, por lo general en un porcentaje ' $30 \%$ de la mezcla total.

El efecto de los tratamientos sobre el crecimiento de las plantas dependió de la especie considerada. La biomasa total de ciprés aumentó con el agregado conjunto de compost y fertilizante, mientras que el roble sólo respondió al agregado de fertilizante. Bioensayos realizados con especies indicadoras (ryegrass) mostraron un incremento de biomasa con agregado de compost de biosólidos tanto en suelos volcánicos disturbados a la misma dosis (Satti 2007) como en suelos de estepa a dosis menores (10 y $20 \mathrm{~g} / \mathrm{kg}$ ) a las utilizadas en nuestro estudio (Laos et al. 2000). En ambos casos, el incremento de biomasa (y de relación biomasa aérea:biomasa radical) fue asociada directamente al aporte de nitrógeno disponible y mineralizable del compost, evidenciando al nitrógeno como el nutriente que más condiciona el crecimiento de ryegrass. En el mismo sentido, un estudio realizado con aplicación de biosólidos mostró mayor crecimiento y acumulación de nutrientes en especies nativas herbáceas de Nueva Zelanda (Gutiérrez-Ginés et al. 2017). Además, en un estudio en el que se agregó nitrógeno en plantines de ciprés que crecían en un suelo característico de bosque de esta especie se detectó mayor relación biomasa aérea:biomasa radical con respecto al control, sólo en un tratamiento con agregado adicional de fósforo (Agüero 2015). La mayor repuesta del ciprés al agregado de compost y nitrógeno mostró que en esta especie no sólo el nitrógeno es limitante. Así, los análisis foliares mostraron que el agregado de compost y $\mathrm{N}$ resultó en una acumulación mayor de $\mathrm{P}$, Ca y Mg en la biomasa aérea (Tabla 4). Ostos et al. (2008) observaron un efecto sinérgico entre la disponibilidad de nitratos y la absorción de Ca y P en plantines de Pistacia lentiscus L. creciendo en un sustrato con $40 \%$ de compost de biosólidos. De manera similar, estudios previos detectaron un aumento de $\mathrm{Mg}$ en el tejido foliar de plantines de ciprés creciendo en un sustrato de suelo y compost de biosólidos (Basil et al. 2009). Además, en nuestro ensayo, el agregado de $\mathrm{N}$ junto con el compost aumentó la biomasa radical del ciprés, lo cual podría favorecer la absorción de los nutrientes aportados por el compost y explicaría la mayor acumulación de nutrientes en las hojas de ciprés. Es decir, que el agregado de N estimularía la absorción de los nutrientes aportados por el compost, lo que resulta en un efecto positivo entre ambos tratamientos. La baja respuesta del ciprés a la adición de $\mathrm{N}$ en forma aislada estaría relacionada con su adaptación a suelos pobres en N (Buamscha et al. 1998) y a una co-limitación con otros nutrientes.

El crecimiento del roble, por el contrario, sólo mostró una fuerte respuesta a la fertilización nitrogenada, lo que indica su papel como limitante del crecimiento. En concordancia, trabajos previos con roble en invernadero utilizando suelos característicos de bosques de esta especie (i.e., suelos ricos en nutrientes) (Diehl et al. 2008), mostraron efectos positivos de la adición de $\mathrm{N}$, incluso a dosis equivalentes a $400 \mathrm{mg} \mathrm{N} / \mathrm{kg}$ suelo, no 
así de la adición de P (Agüero et al. 2014). Este crecimiento notable del roble coincide con las altas tasas fotosintéticas en relación con otros Nothofagus (Varela et al. 2010), mostrando una alta plasticidad fenotípica para aprovechar la disponibilidad de N. La relación biomasa aérea: biomasa radical se modificó con los tratamientos; esta relación indica la asignación de recursos a la parte aérea y subterránea que realizan las plantas, y está determinada genéticamente, pero puede ser alterada por la condición nutricional de la planta y por factores ambientales (disponibilidad de luz y agua), entre otros. Uno de los mecanismos propuestos para explicar la respuesta de las plantas a la fertilización es la capacidad de maximizar la tasa de crecimiento, balanceando la adquisición de recursos aéreos versus subterráneos mediante un compromiso entre la asignación aérea y radical según el nutriente más limitante (Bloom et al. 1985; Marschner 1995). El agregado de compost disminuyó la asignación de recursos de ciprés hacia la biomasa subterránea. El compost aumenta la disponibilidad de $\mathrm{P}$ en el suelo $\mathrm{y}$, por ende, la planta no necesitaría explorar grandes volúmenes de suelo para obtener este nutriente de baja movilidad en el suelo. En cambio, en el roble, solamente la fertilización aumentó la biomasa radical, aunque disminuyó levemente la asignación de recursos hacia las raíces con respecto a la parte aérea. Este aumento en la relación biomasa aérea: biomasa subterránea con la fertilización nitrogenada se reportó previamente en plantines de varias especies forestales (Broncano et al. 1998; Villar-Salvador et al. 2004; Flykt et al. 2008).

\section{Implicancias en las prácticas de restauración}

Estudios previos realizados a campo mostraron que la aplicación de compost mejora la fertilidad de los suelos de bosque de N. pumilio afectados por incendios, pero no contribuye a recuperar la vegetación nativa a corto plazo (Varela et al. 2011). Esto señala la necesidad de implementar otras estrategias de manejo para reinstalar exitosamente especies nativas en sitios disturbados. Nuestros resultados en invernáculo muestran que agregar conjuntamente compost y fertilización nitrogenada mejora de manera significativa el estado nutricional de los plantines de ciprés y modifica levemente la relación biomasa aérea: biomasa subterránea. Por otro lado, aplicar compost a campo podría ser una estrategia efectiva para disminuir la erosión en áreas a restaurar, como se demostró utilizando ramas $(<10 \mathrm{~cm}$ diámetro) y troncos $(<35 \mathrm{~cm}$ diámetro) en sitios quemados (Morales et al. 2013). Finalmente, si bien es importante para la supervivencia a campo de las plantas, para su estado nutricional y para su desarrollo arquitectural, también es necesario tener en cuenta otras variables como la radiación y las condiciones del micrositio donde se instalan las plantas (Gobbi and Schlichter 1998; Kitzberger et al. 2005).

Agradecimientos. Este trabajo fue financiado con fondos provistos por la Agencia Nacional de Promoción Ciencia y Tecnología en el marco del Picto Forestal 2006 №36863. Queremos agradecer a Abel Martínez de la Estación Experimental INTA Bariloche por proveer las plántulas para los ensayos.

\section{Referencias}

Agüero, M. L., J. Puntieri, M. J. Mazzarino, J. Grosfeld, and C. Barroetaveña. 2014. Seedling response of Nothofagus species to $\mathrm{N}$ and P: linking plant architecture to N/P ratio and resorption proficiency. Trees 28:1185-1195. https: //doi.org/10.1007/s00468-014-1029-7.

Agüero, M. 2015. Nutrición temprana en especies arbóreas nativas de alto valor maderable de la región andinopatagónica. Tesis de doctorado. UNComahue Bariloche

Alauzis, M. V., M. J. Mazzarino, E. Raffaele, and L. Roselli. 2004. Wildfires in NW Patagonia: long-term effects on a Nothofagus forest soil. Forest Ecology and Management 192:131-142. https://doi.org/10.1016/j.foreco.2003.11.014.

Ayesa, J., C. López, D. Bran, F. Umaña, and P. Lagorio. 2002. Cartografía biofísica de la Patagonia Norte. En: PAN, PRODESAR. Estación Experimental Agropecuaria Bariloche.

Basil, G., M. J. Mazzarino, L. Roselli, and F. Letourneau. 2009. Efecto del compost de biosólidos en la producción de plantines de Astrocedrus chilensis (Ciprés de la cordillera). Ciencia del Suelo 27:49-55.

Biganzoli, F., T. Wiegand, and W. B. Batista. 2009. Fire-mediated interactions between shrubs in a South American temperate savannah. Oikos 118:1383-1395. https://doi.org/10.1111/j.1600-0706.2009.17349.x.

Bloom, A. J., F. S. I. Chapin, and H. A. Mooney. 1985. Resource limitation in plants - an economic analogy. Annual review of Ecology and Systematics 16:363-392. https://doi.org/10.1146/annurev.es.16.110185.002051.

Broncano, M. J., M. Riba, and J. Retana. 1998. Seed germination and seedling performance of two Mediterranean tree species, holm oak (shape Quercus ilex L.) and Aleppo pine (shape Pinus halepensis Mill.): A multifactor experimental approach. Plant Ecology 138:17-26.

Buamscha, G., M. Gobbi, M. J. Mazzarino, and F. Laos. 1998. Indicators of nitrogen conservation in Austrocedrus chilensis forests along a moisture gradient in Argentina. Forest Ecology and Management 112:253-261. https://doi.org/10.1016/ 
S0378-1127(98)00341-7.

Cellier, A., C. Francou, S. Houot, C. Ballini, T. Gauquelin, and V. Baldy. 2012. Use of urban composts for the regeneration of a burnt Mediterranean soil: A laboratory approach. Journal of Environmental Management 95:5238-5244. https: //doi.org/10.1016/j.jenvman.2010.10.042.

Cooperband, L. R., and J. Middleton. 1996. Changes in chemical, physical and biological properties of cocomposted municipal solid waste (MSW) compost and poultry litter. Compost Science and Utilization 4:24-34. https://doi.org/ 10.1080/1065657X.1996.10701849.

Cordovil, C. M. d. S., A. de Varennes, R. Pinto, and R. C. Fernandes. 2011. Changes in mineral nitrogen, soil organic matter fractions and microbial community level physiological pro'les after application of digested pig slurry and compost from municipal organic wastes to burned soils. Soil Biology and Biochemistry 43:845-852. https://doi.org/ 10.1016/j.soilbio.2010.12.023.

Di Bella, C. M., E. G. Jobbágy, J. M. Paruelo, and S. Pinnock. 2006. Continental fire density patterns in South America. Global Ecology and Biogeography 15:192-199. https://doi.org/10.1111/j.1466-822X.2006.00225.x.

Diehl, P., M. J. Mazzarino, and S. Fontenla. 2008. Plant limiting nutrients in AndeanPatagonian woody species: Effects of interannual rainfall variation, soil fertility and mycorrhizal infection. Forest Ecology and Management 255:29732980. https://doi.org/10.1016/j.foreco.2008.02.003.

Flykt, E., S. Timonen, and T. Pennanen. 2008. Variation of ectomycorrhizal colonisation in Norway spruce seedlings in Finnish forest nurseries. Silva Fennica 42:571-585. https://doi.org/10.14214/sf.234

Gobbi, M., and L. Sancholuz. 1992. Regeneración post-incendio del ciprés de la cordillera (Austrocedrus chilensis) en los primeros años. Bosque 13:25-32. https://doi.org/10.4206/bosque.1992.v13n2-04.

Gobbi, M., and T. Schlichter. 1998. Survival of Austrocedrus chilensis seedlings in relation to microsite conditions and forest thinning. Forest Ecology and Management 111:137-146. https://doi.org/10.1016/S0378-1127(98)00314-4.

Gutiérrez-Ginés, M. J., B. H. Robinson, J. Esperschuetz, E. Madejón, J. Horswell, and R. McLenaghen. 2017. Potential use of biosolids to reforest degraded areas with New Zealand native vegetation. Journal of Environmental Quality 46:906-914. https://doi.org/10.2134/jeq2017.04.0139.

Kitzberger, T., E. Raffaele, K. Heinemann, and M. J. Mazzarino. 2005. Effects of fire severity in a north Patagonian subalpine forest. Journal of Vegetation Science 16:5-12. https://doi.org/10.1111/j.1654-1103.2005.tb02333.x.

Kowaljow, E., and M. J. Mazzarino. 2007. Soil restoration in semiarid Patagonia: Chemical and biological response to different compost quality. Soil Biology and Biochemistry 39:1580-1588. https://doi.org/10.1016/j.soilbio.2007.01.008.

Kuo, S. 1996. Phosphorus. Pp. 869-919 in D. L. Sparks, A. L. Page, P. A. Helmke, R. H. Loeppert, P. N. Soltanpour, M. A. Tabatabai, C. T. Johnston and M. E. Sumner (eds.). Methods of Soil Analysis. Part 3. Chemical methods. Soil Science Society of America, Madison, WI, USA.

La Manna, L., and C. Barroetaveña. 2011. Propiedades químicas del suelo en bosques de Nothofagus antarctica y Austrocedrus chilensis afectados por fuego. Revista de la Facultad de Ciencias Agrarias Universidad Nacional de Cuyo 43:41-55.

Laos, F., P. Satti, I. Walter, M. J. Mazzarino, and S. Moyano. 2000. Nutrient availability of composted and noncomposted residues in a Patagonian Xeric Mollisol. Biology and Fertility of Soils 31:462-469. https://doi.org/ 10.1007/s003740000192.

LeBauer, D. S., and K. K. Treseder. 2008. Nitrogen limitation of net primary productivity in terrestrial ecosystems is globally distributed. Ecology 89:371-379. https://doi.org/10.1890/06-2057.1.

Marschner, H. 1995. Mineral nutrition of higher plants. Academic Press, London.

Morales, D., C. M. Rostagno, and L. La Manna. 2013. Runoff and erosion from volcanic soils affected by fire: the case of Austrocedrus chilensis forests in Patagonia, Argentina. Plant and Soil 370:367-380. https://doi.org/10.1007/s11104013-1640-1.

Oddi, F. J. 2013. Los incendios y la dinámica de Fabiana imbricata en el noroeste de la Patagonia a escala de paisaje. Su relación con los factores ambientales y el uso del suelo. Universidad Nacional del Comahue, Bariloche, Río Negro, Argentina.

Ostos, J. C., R. López-Garrido, J. M. Murillo, and R. López. 2008. Substitution of peat for municipal solid waste- and sewage sludge-based composts in nursery growing media: Effects on growth and nutrition of the native shrub Pistacia lentiscus L. Bioresource Technology 99:1793-1800. https://doi.org/10.1016/j.biortech.2007.03.033.

Parfitt, R. L., D. J. Ross, D. A. Coomes, S. J. Richardson, M. C. Smale, and R. A. Dahlgren. 2005. N and P in New Zealand soil chronosequences and relationships with foliar N and P. Biogeochemistry 75:305-328. https://doi.org/10.1007/ s10533-004-7790-8.

Satti, P. 2007. Biodisponibilidad de fósforo y nitrógeno en suelos volcánicos bajo bosque nativo, disturbados y enmendados. Universidad Nacional del Comahue.

Schlesinger, W. H. 1997. Biogeochemistry: an analysis of global change. Academic Press, San Diego, USA.

Tognetti, C., F. Laos, M. J. Mazzarino, and M. T. Hernández. 2005. Composting vs vermicomposting: a comparison of the end product quality. Compost Science and Utilization 13:6-13. https://doi.org/10.1080/1065657X.2005.10702212.

Torres Curth, M. I., L. Ghermandi, and C. Biscayart. 2012. Are Fabiana imbricata shrublands advancing over northwestern Patagonian grasslands? A population dynamics study involving 're and precipitation. Journal of Arid Environments 83:78-85. https://doi.org/10.1016/j.jaridenv.2012.03.011.

Urretavizcaya, M. F. 2010. Propiedades del suelo en bosques quemados de Austrocedrus chilensis en Patagonia, Argentina. Bosque 31:140-149. https://doi.org/10.4067/S0717-92002010000200007. 
Urretavizcaya, M. F., and G. Defossé. 2004. Soil seed bank of Austrocedrus chilensis (D. Don) Pic. Serm. et Bizarri related to different degrees of fire disturbance in two sites of southern Patagonia, Argentina. Forest Ecology and Management 187:361-372. https://doi.org/10.1016/j.foreco.2003.07.001.

Urretavizcaya, M. F., P. L. Peri, L. Monelos, H. Arriola, M. F. Oyharçabal, L. Contardi, M. Muñoz, E. Sepúlveda, and G. Defosse. 2018. Condiciones de suelo y vegetación en tres bosques quemados de Nothofagus pumilio en Argentina y experiencias para su restauración activa. Ecología Austral 28:383-399. https://doi.org/10.25260/EA.18.28.2.0.480.

Varela, S. A., M. Gobbi, and F. Laos. 2011. Can biosolids compost improve, in the short term, native vegetation and soils fertility in burned Nothofagus pumilio forest in Patagonia, Argentina? Bosque 32:267-278.

Varela, S. A., M. E. Gobbi, and F. Laos. 2006. Banco de semillas de un bosque quemado de Nothofagus pumilio: efecto de la aplicación de compost de biosólidos. Ecología Austral 16:63-78. https://doi.org/10.4067/S0717-92002011000300008.

Varela, S. A., and A. Martínez. 2013. Uso del compost de biosólidos en la formulación de sustratos para la producción industrial de plantas de Nothofagus alpina. Bosque 34:281-289. https://doi.org/10.4067/S0717-92002013000300004.

Veblen, T. T., T. Kitzberger, and A. Lara. 1992. Disturbance and forest dynamics along a transect from Andean rain forest to Patagonia shrubland. Journal of Vegetation Science 3:507-520.

Veblen, T. T., C. Donoso, T. Kitzberger, and A. J. Rebertus. 1996. Ecology of southern Chilean and Argentinean Nothofagus forests. Pp. 293-353 en T. T. Veblen, R. S. Hill and J. Read (eds.). The ecology and biogeography of Nothofagus forests. Yale University Press. New Haven. https://doi.org/10.2307/3235807.

Villar-Salvador, P., R. Planelles, E. Enrýìquez, and J. P. Rubira. 2004. Nursery cultivation regimes, plant functional attributes, and field performance relationships in the Mediterranean oak Quercus ilex L. Forest Ecology and Management 196:257-266. https://doi.org/10.1016/j.foreco.2004.02.061. 
